\title{
Generalists and specialists along a latitudinal transect: patterns of thermal adaptation in six species of damselflies
}

\author{
Viktor Nilsson-Örtman, ${ }^{1,4}$ Robby Stoks, ${ }^{2}$ Marjan De Block, ${ }^{2}$ and Frank Johansson ${ }^{1,3}$ \\ ${ }^{1}$ Department of Ecology and Environmental Science, Umeå University, SE-90187 Umeå, Sweden \\ ${ }^{2}$ Laboratory of Aquatic Ecology and Evolutionary Biology, University of Leuven, Ch. Deberiotstraat 32, BE-3000 Leuven, Belgium \\ ${ }^{3}$ Department of Ecology and Genetics, Uppsala University, SE-75236 Uppsala, Sweden
}

\begin{abstract}
Tropical organisms colonizing temperate environments face reduced average temperatures and dramatic thermal fluctuations. Theoretical models postulate that thermal specialization should be favored either when little environmental variation is experienced within generations or when among-generation variation is small relative to within-generation variation. To test these predictions, we studied six temperate species of damselflies differing in latitudinal distribution. We developed a computer model simulating how organisms experience environmental variation (accounting for diapause and voltinism) and performed a laboratory experiment assaying thermal sensitivities of growth rates. The computer model showed opposing latitudinal trends in among- and within-generation thermal variability: within-generation thermal variability decreased toward higher latitudes, whereas relative levels of among-generation thermal variability peaked at midlatitudes (where a shift in voltinism occurred). The growth experiment showed that low-latitude species were more thermally generalized than mid- and high-latitude species, supporting the prediction that generalists are favored under high levels of within-generation variation. Northern species had steeper, nearexponential reaction norms suggestive of thermal specialization. However, they had strikingly high thermal optima and grew very slowly over most of the thermal range they are expected to experience in the field. This observation is at present difficult to explain. These results highlight the importance of considering interactions between life history and environmental variation when deriving expectations of thermal adaptation.
\end{abstract}

Key words: Coenagrion spp.; damselflies; developmental plasticity; environmental variability; growth rate; life history; optimality theory; reaction norm; thermal sensitivity; thermal variability.

\section{INTRODUCTION}

In order to survive and reproduce, most organisms must be able to perform key physiological functions such as growth, locomotion, and feeding over a wide range of environmental conditions. In this respect, temperature is of particular interest as it affects all biological rate processes, from biochemical kinetics to rates of evolution (Kingsolver 2009). Current research on thermal adaptation largely focuses on how thermal sensitivities of different traits evolve in response to selection imposed by varying thermal regimes. Thermal performance curves (TPCs) are used to describe how the expression of a trait varies as a function of temperature. Comparative studies across all taxonomic levels have shown that TPCs share several common features: a more or less gently increasing "rise" phase, a single optimum, and a rapidly decreasing "fall" phase above the optimum. Additionally, these features vary in certain predictable ways (Navas 1996, Izem and Kingsolver 2005, Dell et al. 2011), suggesting that the shape of TPCs

Manuscript received 21 October 2011; accepted 23 November 2011; final version received 2 January 2012. Corresponding Editor: S. J. Simpson.

${ }^{4}$ E-mail: viktor.nilsson@emg.umu.se is under direct selection. A species can be considered a thermal generalist if high performance is maintained over a wide range of temperatures (the rise and fall phases having shallow slopes), and a thermal specialist if high performance is restricted to a narrow range of temperatures (the rise and fall phases having steeper slopes) (Angilletta 2009, Dell et al. 2011).

Two optimality models in particular have explored how generalists and specialists evolve in response to environmental variation. Both models share an assumption that there is a trade-off between maximum performance and performance breadth, but differ in their assumptions about how performance affects fitness. Gilchrist (1995) modeled the evolution of a performance trait having an additive effect on fecundity (an individual's fitness was equal to the sum of performance over its life span). High growth rates could have this effect on fitness if fast-growing genotypes manage to complete more generations within a given time span (Roff 2002). Lynch and Gabriel (1987), on the other hand, modeled the dynamics of a trait related to fitness through its effect on survival (an individual's fitness was equal to the geometric mean of performance over its life span). This is possible for growth rates if, for example, predation or winter mortality is strongly size 
dependent (Arendt 1997). These models produced two main testable predictions. First, both models predicted that the thermal optimum should match the mean (or modal) environmental temperature. However, the two models differed dramatically regarding their second prediction, relating to performance breadth. Lynch and Gabriel's (1987) model predicted that the environment experienced by an individual during its lifetime has the greatest influence on performance breadth; in highly variable environments thermal generalists should always be favored. Gilchrist's (1995) model, on the other hand, predicted that the relative amount of thermal variability among and within generations will be the main determinant of performance breadth and that a specialist always has higher fitness than a generalist unless the amount of variation among generations is large relative to that within generations.

To test these predictions, we investigated the thermal sensitivity of larval growth rates in six damselfly species (Odonata: Coenagrionidae) distributed along a latitudinal gradient. Because growth rates can be argued to contribute to fitness by affecting both fecundity and survival, they are especially interesting in this context. Growth rates are known to display predictable geographic variation in odonates (Van Doorslaer and Stoks 2005a, De Block et al. 2008, Stoks and De Block 2011) as well as other taxa (e.g., Demont et al. 2008, Pöykkö and Tammaru 2010). The two optimality models were intended to describe intraspecific variation in performance breadth. Inter- and intraspecific studies of TPC variation have sometimes reached different conclusions, for example regarding the amount of variation in the slope of the temperature dependence of metabolic rates (Brown et al. 2004, Irlich et al. 2009, Dell et al. 2011), indicating that some caution may be warranted. However, several features of TPC variation remain remarkably similar across different traits and hierarchical levels (Dell et al. 2011). We therefore see no strong reason why the hypotheses generated by these models cannot be transferred to interspecific comparisons as long as each species is tested at multiple temperatures. Note however that we thereby make the assumption that species have evolved locally optimal performance breadths.

Our first step was to quantify among- and withingeneration thermal variability experienced by these species in nature, taking variation in generation length and diapause into account. The importance of both factors has been suggested previously (Angilletta et al. 2006), but they have rarely been considered in empirical studies (for two notable exceptions, see Ragland and Kingsolver [2007] and Cooper et al. [2010]), and never jointly. We then investigated the thermal sensitivity of growth rates in a common-garden experiment of the same species. Assuming that species having similar distributions should respond to changes in temperature in a common way (because they inhabit similar environments), we predicted that the thermal sensitivity of these traits should correlate with either the amount of within-generation thermal variability or with the ratio of among-to-within generation thermal variability.

\section{Methods}

\section{The study species}

Six congeneric damselfly species were included in this study, chosen because they clearly differ in the latitudinal extent of their distribution ranges: two species are exclusively found in Southern Europe (Coenagrion mercuriale, C. scitulum; see Plate 1), two species have large ranges spanning much of Central Europe (C. puella and C. pulchellum), and two species are exclusively found in Northern Europe (C. armatum, C. johanssoni) (Fig. 1).

It is well known that dragonflies show highly plastic life histories, not only among species but also among populations (Corbet et al. 2006). The northern species in this study are semivoltine in the core part of their range (requiring two years to finish larval development), whereas central species are univoltine (Norling 1984, Corbet 1999). The life cycles of southern species are poorly known, but phenological data (showing a spring peak and a late summer peak in abundance) may suggest a mixed strategy, where at least part of the population complete two generations per year (Dijkstra and Lewington 2006; J. Garcia, personal communication). This increase in generation length toward higher latitudes is typical of odonates (Norling 1984, Corbet et al. 2006).

\section{Estimating thermal variation within and among generations}

To derive detailed predictions from the two optimality models, we wanted to know how temperature variation is expressed among and within generations. For this purpose we developed a computer model that simulates the life cycle of damselfly larvae based on information on seasonal variation in water temperature. The model is briefly summarized next (for a full description and $\mathrm{R}$ code see Appendix A and the Supplement).

We first defined the length of a generation as the period required to accumulate 4344 degree-days (based on data from six Coenagrion species reared from egg to emergence by V. Nilsson-Örtman and S. Śniegula [unpublished data]). The computer model then recursively calculated the number of accumulated degree-days at 12-h intervals (night and day temperatures) until the required number of degree-days had been reached. To ensure realism, it was assumed that emergence could only occur between 1 May and 31 August; if the required number of degree-days were reached outside of this period, emergence was postponed to 1 May the next year. Thus, the computer model simultaneously simulated the temporal extent of each generation and the thermal environment it had experienced. We performed a total of 18 model runs with environmental data corresponding to conditions in areas within the core of 
Sampled and climatically modeled $\square$ Climatically modeled
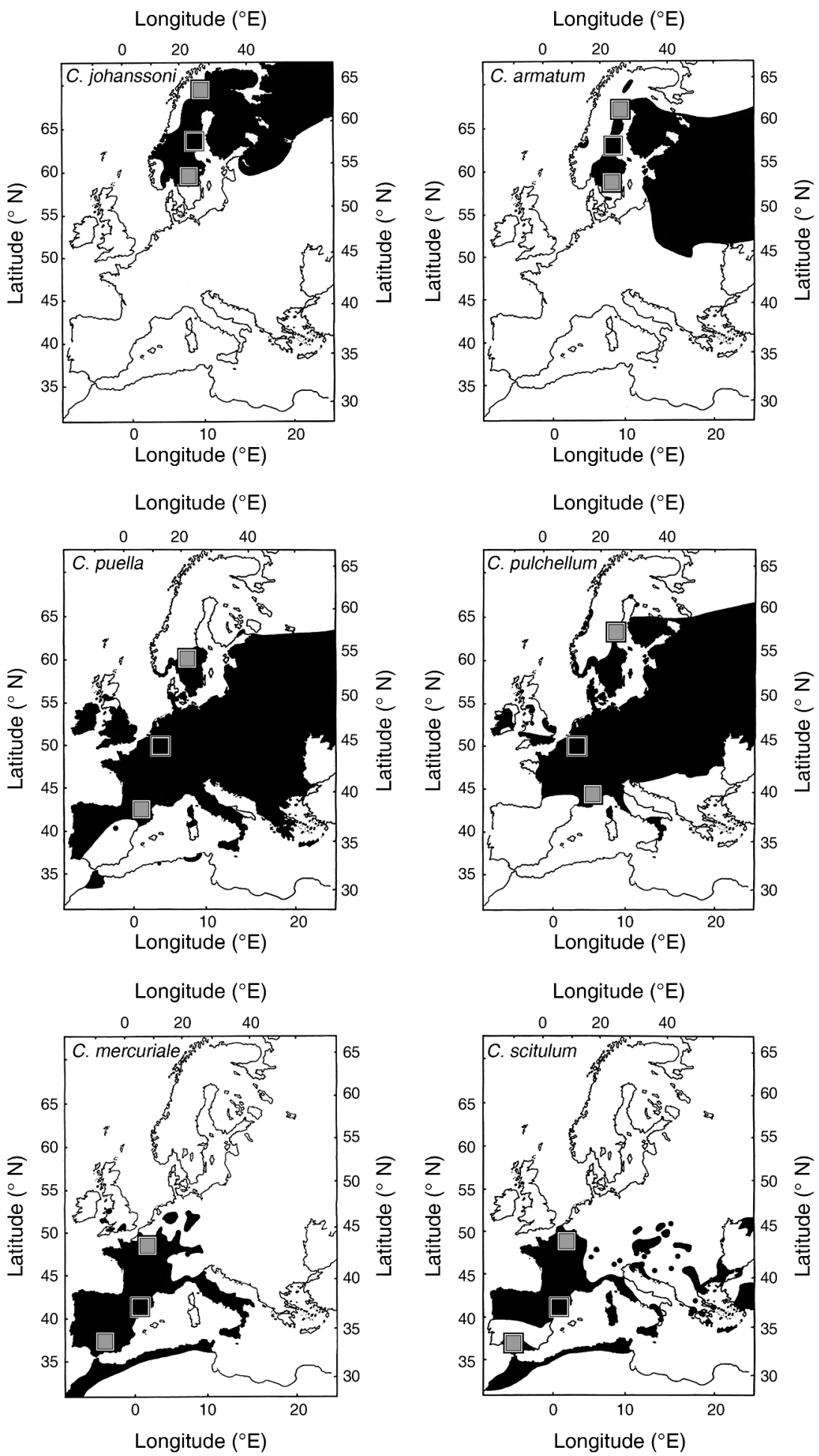

FIG. 1. European distribution of the six Coenagrion damselfly species used in this study. Maps are reproduced from Askew (2004) with permission from Apollo Books and updated with information from Dijkstra and Lewington (2006). The black and gray boxes represent areas from which we extracted the meteorological forcing data, which we used to simulate surface water temperatures and estimate among- and within-generation thermal variability. Black boxes represent the locations of the central populations for which we have estimated both patterns in thermal variability and TPCs of larval growth rates (corresponding to solid black circles in Fig. 3); gray boxes represent areas that were only used to investigate patterns in thermal variability (corresponding to open circles in Fig. 3). 
each species' range, as well as close to the southern and northern range margin (see the black and gray squares in Fig. 1 for orientation).

A priori knowledge on the thermal biology of damselflies motivated the inclusion of a diapause threshold (a mechanism by which development is retarded in autumn, ensuring that the cold season is endured in the resistant larval stage) in the model. Temperate odonates enter diapause at temperatures below $8-10^{\circ} \mathrm{C}$ (Corbet 1999), and therefore periods of temperatures below $8^{\circ} \mathrm{C}$ were assumed to have no effect on the thermal variation experienced by growing larvae (Angilletta et al. 2006, Cooper et al. 2010). Exposures to very low temperatures may also be important, by affecting survival, but available information regarding the overwintering ecology of odonates is too scarce to incorporate the effects of subzero temperatures in our model. We do know, however, that Coenagrion species are freeze-tolerant and several species regularly spend several months completely frozen into ice (Corbet 1999:182), suggesting that variation in subzero temperatures may be of less importance.

Environmental data needed to run the life-cycle simulations were generated by modeling realistic surface water temperatures in small, shallow water bodies at different latitudes (the typical habitat of these species) using the lake model FLake (Lake Model FLake 2009). The following meteorological observations were used as input data: surface thermal radiation, solar radiation, wind speed, dewpoint temperature, and air temperature. The input data were extracted from the ECMWF EraInterim data set (Simmons et al. 2007) from the years 1999-2009 at 12-h intervals (night and day). To evaluate the results from the FLake simulations, we compared them against real measurements extracted from a database of Swedish lakes $(N=109)$ provided by the Swedish University of Agricultural Sciences (available online $)^{5}$ and through performing a literature search for temperature data from shallow, central European lakes $(N=9$; Appendix A).

The life-cycle simulations were used to calculate the following statistics (based on 20 simulated generations): (1) average temperature; (2) among-generation thermal variation (defined as the median absolute deviation [MAD] of the generation mean temperature relative to the grand mean for all generations); and (3) withingeneration thermal variation (defined as the MAD of the generation mean, averaged across generations). We also calculated the ratio of among- to within-generation variability. The same statistics were calculated among and within years, to investigate the effect of variation in voltinism. The relationships between the different measures of variation and latitude were investigated using one-way ANOVAs including the quadratic and cubic latitude terms prior to simplification. The choice

${ }^{5}$ http://webstar.vatten.slu.se/db.html of variability measure did not alter the qualitative outcome of the simulations, as trial runs using the coefficient of variation and SD all gave the same results.

\section{Laboratory assay of growth rates}

Mated females were collected from three localities from within the core part of each of the six species' ranges (three localities were used to minimize the influence of maternal and environmental effects). Bad weather prevented sampling of more than two populations of $C$. johanssoni. A list of all sampled populations and the number of families are given in Appendix E: Table E1. Because of time, space, and labor constraints, rearing was divided between two laboratories and over two years: C. armatum, $C$. puella, and $C$. johanssoni were collected and reared during 2008 and $C$. mercuriale, $C$. pulchellum, and $C$. scitulum during 2009. One species in each pair of northern and southern species was reared in Leuven and the other species in Umeå in order to avoid confounding laboratory rearing conditions with latitude. Females of $C$. pulchellum and $C$. armatum were brought to the laboratory in Umeå by car to oviposit; eggs from the other species were sent by air mail to the laboratory in Umeå, Sweden (Coenagrion puella and C. scitulum) or Leuven, Belgium (C. johanssoni and C. mercuriale) in 50$\mathrm{mL}$ plastic tubes containing $\sim 30 \mathrm{~mL}$ of non-chlorinated water (the excess air providing oxygen for the embryos). After hatching, five full-sib offspring from the between two and 12 females from each locality (median sample size was six clutches) were reared in each of four temperatures: $16.3^{\circ}, 19.5^{\circ}, 21.5^{\circ}$, and $24.0^{\circ} \mathrm{C}\left(24.0^{\circ} \mathrm{C}\right.$ added during the 2009 rearing). Larvae were reared individually under a 14:10 light: dark (L:D) light regime and fed six days a week on laboratory-reared brine shrimp, Artemia sp., nauplii ad libitum (282 \pm 62 nauplii, mean $\pm \mathrm{SD}, n=25$ food doses). Rooms were switched randomly on three occasions during the rearing to avoid room effects. For further details on the rearing see Appendix B.

On day 0 , the experiment consisted of 2002 individual larvae, representing 119 full-sib families. These families constitute the replicate unit of the experiment. Larvae were photographed at day $0,42,84$, and 126 , and the maximum distance between the distal parts of the eyes was calculated from these photographs. Head width is a good approximation of overall size in dragonflies (Corbet 1999). Image analyses were carried out using ImageJ 1.43 (National Institutes of Health, Bethesda, Maryland, USA) and reference photographs of a glass scale were used for calibration. Missing data from individuals lacking data from a single measurement event in a given temperature were imputed based on data from families with complete data (Appendix C). Individuals lacking two or more measurement events were excluded. In total we ended up analyzing data from 1419 individual larvae representing 101 families.

We adopted an approach to calculate growth rates proposed by Rose et al. (2009) and recently applied to 


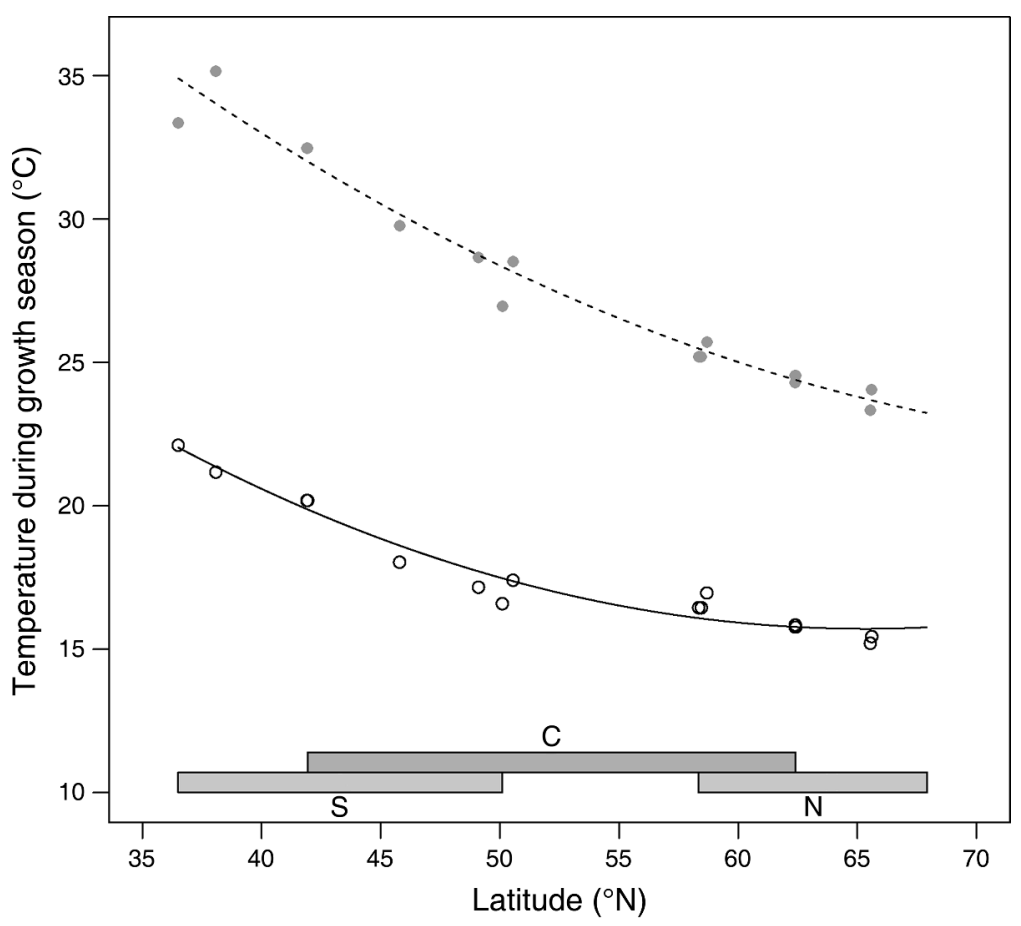

FIG. 2. Average (open circles) and maximal (solid gray circles) temperatures at representative sites from the southern, central, and northern part of the range of each of the six investigated damselfly species. Data come from the lake model FLake simulation of surface water temperature based on climate data for radiation fluxes, wind speed, wet bulb temperature, and air temperature. Average and maximal temperatures are shown for each modeled site (see Fig. 1 for orientation). The lines show the cubic regressions of average temperature (solid curve) above $8^{\circ} \mathrm{C}$ and maximal temperature (dashed curve) during the 11-year period investigated. See Results for test statistics. The latitudinal range of the southern (S), central (C), and northern (N) species in this study is shown as gray horizontal bars just above the $x$-axis.

Coenagrion species (Śniegula et al., in press), by modeling the growth trajectories of the individuals from a family at a given temperature as a third-degree polynomial function with head width as a function of time. From this we calculated size-corrected relative growth rates (RGRs) as the slope of the curve at the point in time when one-fifth of the adult size (based on field-collected adults) had been reached (Appendix D).

\section{Statistical analysis of reaction norms}

We used linear mixed-effect models (LME) to test for shape differences in the reaction norms of size-corrected larval RGRs. Because all experimental temperatures were found to lie within the initial, rising part of TPCs, it was crucial to test for significant differences in curvature, as the shape of the rising phase is closely connected to the generalist/specialist dichotomy (Dell et al. 2011, Huey and Kingsolver 2011). We performed two separate analyses to investigate differences among groups of species (i.e., northern, central, and southern) as well as among species. Significance levels of random effects were estimated by likelihood ratio tests, but variations at these levels are not within the scope of this paper. Although maternal effects have been found to be negligible in coenagrionids (Strobbe and Stoks 2004, Shama et al. 2011), it should be noted that nonadditive and maternal effects may be part of the (random) family effect.

In the first analysis, we treated latitude-of-origin (group of species), temperature, and the interaction between latitude-of-origin $\times$ temperature and latitudeof-origin $\times$ temperature $^{2}$ as fixed effects, whereas species (nested in latitude of origin), population (nested in species), and family (nested in population) were treated as random factors. The quadratic temperature term was included to test for significant, nonlinear relationships.

In the second analysis, species were treated as a fixed effect together with temperature and the interactions between species $\times$ temperature and species $\times$ temperature $^{2}$. Population (nested in species) and family (nested in population) were treated as random factors. All statistical analyses were carried out using R 2.10.1 (R Development Core Team 2008).

\section{Results}

\section{Thermal variability among and within generations}

As expected, the FLake simulations showed that average and maximal temperatures decreased from south to north (Fig. 2), matching the gradient in generation length. The relationship for average temperatures was nonlinear, as the latitude $^{2}$ effect was 

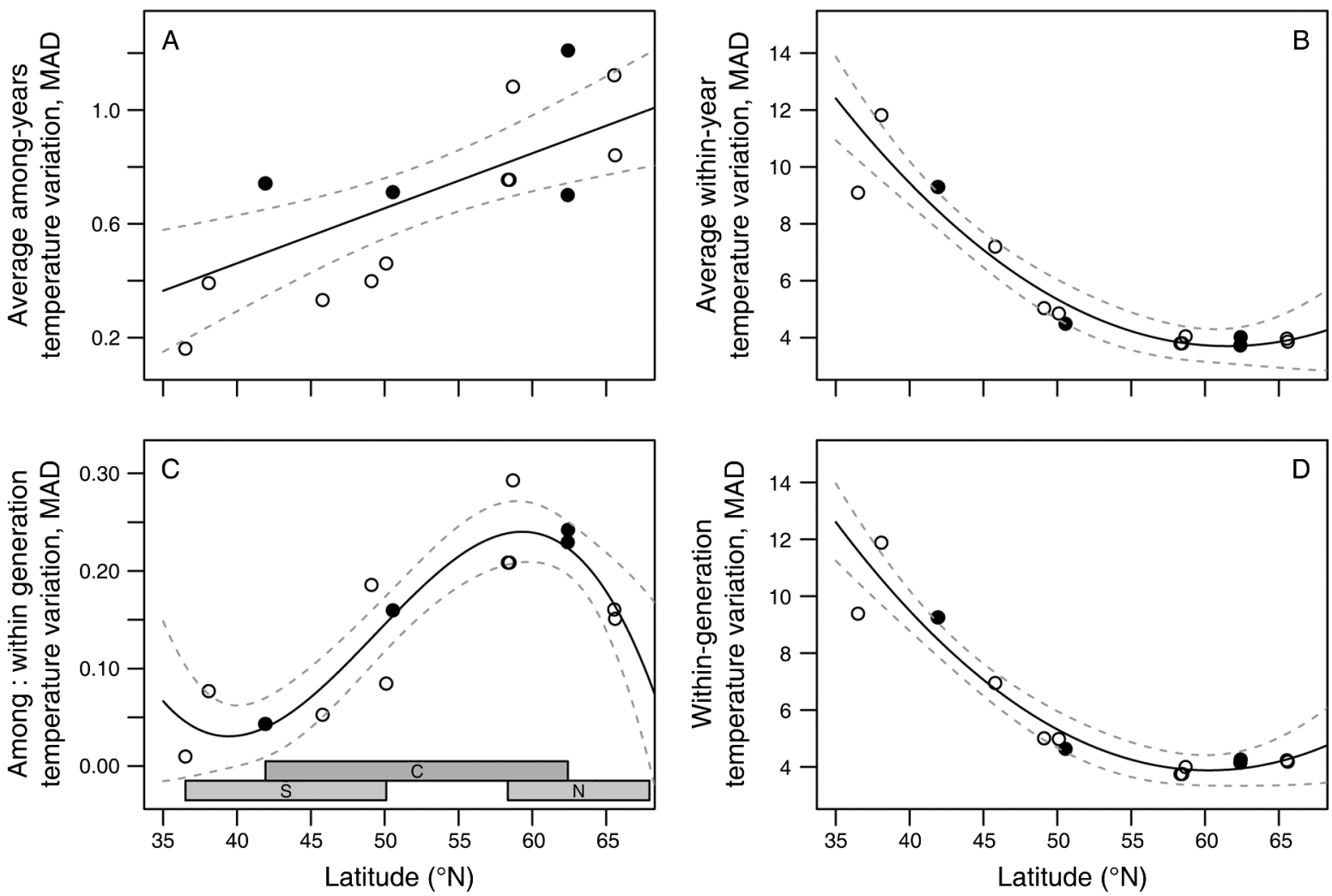

FIG. 3. Results from the lake model FLake simulation of surface water temperature at representative sites from the southern, central, and northern part of the range of each of the six investigated species, showing latitudinal patterns in thermal variability as MAD, median absolute deviation. The top row shows variation (A) among and (B) within years. Variation among years is the median absolute deviation of the grand mean across all 11 yearly means. Within-year variation is calculated as the mean of the median absolute deviation around the yearly means. The bottom row shows how environmental variation is expressed (C) among and (D) within generations based on the simulations of generation length. Among-generation variation is the median absolute deviation of the grand mean across the 20 simulated generations. Within-generation variation is the mean of the median absolute deviation around the generation mean. Temperatures below $8^{\circ} \mathrm{C}$ were excluded from the calculations because diapause is initiated below this temperature. Note that, because among-generation variation is influenced by generation length, which increases toward higher latitudes, it shows a hump-shaped pattern, reaching a maximum at $\sim 58^{\circ} \mathrm{N}$, above which a semivoltine life cycle dominates. Symbols denote regions from which the laboratory-reared populations were sampled (solid black circles) and regions that were only climatically modeled (open circles). Solid lines show best-fitting polynomial regressions up to the third order (if higher-order terms were found significant). Dashed lines indicate $95 \%$ CI of the regression parameters. The latitudinal ranges of the southern (S), central (C), and northern (N) species in this study are shown as gray horizontal bars in panel (C). Note that the solid black circles overlap for south and central species.

significant in the ANOVA $\left(F_{1,15}=24.77, P<0.001\right)$ and maximal temperature showed the same pattern $\left(F_{1,15}=\right.$ 7.51, $P=0.015)$. Looking at thermal variation among and within years (accounting for diapause, but ignoring variation in voltinism) we found that the among-year thermal variation increased linearly toward higher latitudes (Fig. 3A) $\left(F_{1,16}=14.138, P=0.002\right)$. Within years, the pattern was the opposite; variation around the yearly mean decreased with latitude $\left(F_{1,15}=127.0, P<\right.$ 0.001 ), and this relationship was nonlinear (latitude ${ }^{2}$ term significant; $\left.F_{1,15}=18.53, P<0.001\right)$ (Fig. 3B). In an obligately univoltine organism (with diapause), the patterns in Fig. 3A and B would translate directly into within- and among-generation thermal variability. The same patterns were also observed in the empirical data set (Appendix E: Fig. E2).
In the computer model of larval life cycles, all populations below $46^{\circ} \mathrm{N}$ (southern and central populations of southern species and southern populations of central species) were obligately univoltine. At around $50^{\circ} \mathrm{N}$ (northern populations of southern species and central populations of central species), one or two in 10 generations required more than one year, marking the southernmost limit of a semivoltine life cycle. At $58^{\circ} \mathrm{N}$ (northern populations of $C$. puella and southern populations of northern species), a semivoltine life cycle was dominating. Above $62^{\circ} \mathrm{N}$ (central populations of northern species and northern populations of $C$. pulchellum) a generation took 2-3 years to finish, whereas at $68^{\circ} \mathrm{N}$ (northern populations of northern species), a generation took 3-4 years.

When voltinism patterns are taken into account, patterns of thermal variation change from those 

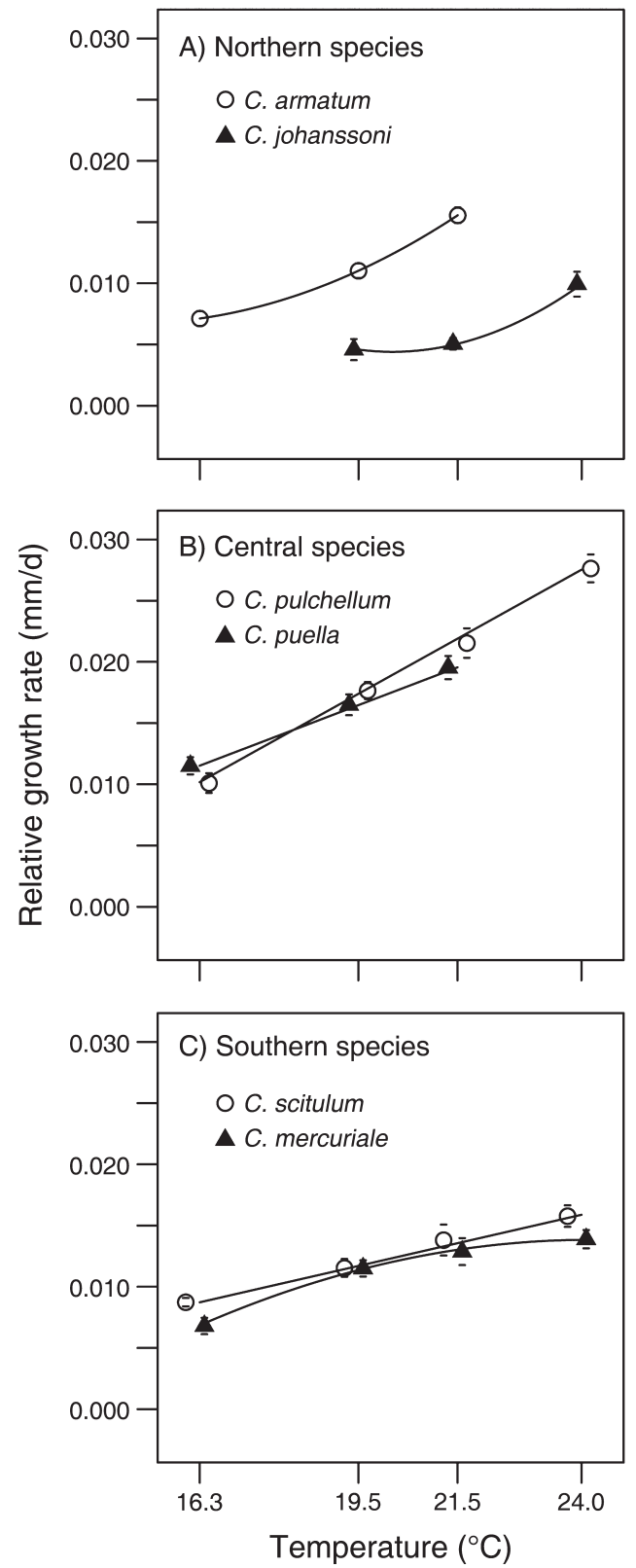

FIG. 4. Thermal reaction norms of larval growth rates of six Coenagrion damselfly species, representing (A) northern, (B) central, and (C) southern European distributions. Vertical bars (some hidden by symbols) show 95\% CI around the mean. Fitted curves include either linear or quadratic temperature terms, depending on whether these variables were significant as judged by separate ANOVAs.

observed among years. The ratio of among-to-within generation thermal variation displayed a hump-shaped relationship with latitude, reflected by a significant latitude $^{3}$ term in the ANOVA (MS $=0.016, F_{1,14}=$ 13.92, $P=0.002$; Fig. 3C). Within generations, the pattern remains very close to temperature variation within years (cf. Fig. 3B, D).

\section{Thermal response of species differing in latitudinal distribution}

Despite that average surface water temperatures were estimated to range between $14^{\circ}$ and $22^{\circ} \mathrm{C}$ across the entire range of these species (Fig. 2), few families showed any tendency for reduced growth rates even at the highest experimental temperature (Fig. 4; Appendix E: Fig. E2), suggesting that maximal growth rates of most families are reached at or above $21.5-24^{\circ} \mathrm{C}$ in all species.

In the LME model with a fixed latitude-of-origin effect and random species effects, growth rates of damselfly larvae increased with increasing temperature (the temperature effect; Table 1A). The latitude-oforigin term was not significant. However, groups of species differing in latitudinal distribution differed in both slope and curvature, as latitude-of-origin showed significant interactions with both temperature and temperature $^{2}$ (Table 1A). The latitude-of-origin $\times$ temperature interaction was caused by a steeper slope of the central species (growing increasingly faster than the two other groups of species with increasing temperature) and the latitude-of-origin $\times$ temperature $^{2}$ effect was only evident in the group of southern species, displaying a slight upward-convex curvature. The group of northern species, however, displayed large variation when analyzed together, indicating large differences either within or among species. These patterns are obscured when species are analyzed together, leading us to the detailed exploration of variation among species.

In the LME model with fixed species effect, all fixed effects and interactions between them were significant, indicating divergence in height, slope, and curvature among species (Table 1B). The reaction norms of individual species are shown in Fig. 4, with speciesspecific regression lines including linear or quadratic temperature terms, depending on whether these variables were significant as judged by separate ANOVAs (Appendix E: Table E2). The two northern species displayed upward-concave reaction norms within the investigated thermal range, the slope of which were flattest at low temperatures, but became steeper as the temperature increased (Fig. 4A). Reaction norms of both central species, on the other hand, displayed a steep, linear slope over the entire temperature range (Fig. 4B), while reaction norms of southern species were markedly flat overall, either linear (C. scitulum) or steepest at lower temperatures, flattening out as temperature increased (C. mercuriale) (Fig. 4C).

\section{DisCusSION}

We investigated the thermal sensitivity of larval growth rates of Coenagrion species distributed at three different latitudes in order to test discrepancies between two optimality models on the evolution of specialists and generalists. We hypothesized that species having similar distributions should respond to changes in temperature in a common way and that thermal reaction norms of growth rates would correlate with either the 
TABLE 1. Results from the LME models on the relative growth rates of damselflies, either (A) including a fixed latitude-of-origin effect and random species effects or (B) considering species as fixed effects.

\begin{tabular}{llrrr}
\hline \multicolumn{1}{c}{ Effect } & \multicolumn{1}{c}{$\mathrm{df}$} & \multicolumn{1}{c}{$\chi^{2}$} & \multicolumn{1}{c}{$P$} \\
\hline A) With fixed latitude-of-origin effect & & & & \\
Intercept & 1,269 & 92.19 & & $<0.0001$ \\
Temperature & 1,269 & 1607.98 & & $<0.0001$ \\
Latitude-of-origin & 2,3 & 4.61 & & 0.1215 \\
Latitude-of-origin $\times$ temperature & 2,269 & 109.68 & & $<0.0001$ \\
Latitude-of-origin $\times$ temperature & 3,269 & 6.43 & 25.43 & $<0.0003$ \\
Species(latitude-of-origin) & 1 & & 1.42 & 0.2333 \\
Population(species) & 1 & & 10.66 & 0.0011 \\
Family(population) & 1 & & & \\
B) With fixed species effect & & & & $<0.0001$ \\
Intercept & 1,263 & 8154.94 & & $<0.0001$ \\
Temperature & 1,263 & 1332.91 & & $<0.0001$ \\
Species & 5,11 & 134.54 & & $<0001$ \\
Species $\times$ temperature & 5,263 & 15.18 & & 0.0001 \\
Species $\times$ temperature & 6,263 & 6.51 & & $<.73$ \\
Population(species) & 1 & & 15.28 & $<0.0001$ \\
Family(population) & 1 & & & \\
\hline
\end{tabular}

Note: The latitude-of-origin term in panel A corresponds to groups of species (i.e., northern, central, and southern species) differing in latitudinal distributions.

amount of within-generation thermal variability or with the ratio of among-to-within generation thermal variability they experience.

\section{Predictions from the life-cycle simulations}

Based on the computer simulations, we found opposing latitudinal trends (Fig. 3) in among- and within-generation thermal variability. The shapes of these relationships were set by the interaction between diapause, generation length, and thermal variability among and within years. Relative levels of amonggeneration thermal variability (Fig. 3C) peaked at midlatitudes. The cause behind this was that although among-year variation increased with latitude (Fig. 3A), a shift in voltinism occurred around $58^{\circ} \mathrm{N}$ and the progressively longer life spans above this latitude acted to average out the among-year variation. Withingeneration thermal variation on the other hand showed a monotonically decreasing latitudinal trend (Fig. 3D), which was caused by two factors: (1) including the effect of the temperature-induced diapause by assuming that temperatures below the diapause threshold do not contribute to the thermal environment experienced by growing larvae; and (2) that, because high-latitude environments display more rapid shifts from cold winter temperatures to warm summer temperatures, relatively less time is spent at intermediate temperatures. This pattern was evident in both the simulated and empirical data sets (Appendix E: Fig. E1). Therefore, Lynch and Gabriel's (1987) model predicts that species should become increasingly specialized toward higher latitudes (within-generation thermal variability decreases; Fig. 3D), whereas Gilchrist's (1995) model conversely predicts that species should become increasingly generalized (among-to-within generation thermal variability increase up to where the sampled populations of northern species are located; Fig. 3C). We also expect that the thermal optima should match the mean temperature of the environment.

\section{General patterns of growth rates}

Species having similar distributions responded to temperature in a similar way (Fig. 4; Appendix E: Fig. E1). Central species grew faster than southern species at all experimental temperatures (Fig. 4B). This pattern is consistent with a scenario where central species compensate for the shorter growth season at higher latitudes through adaptive countergradient variation (Conover and Schultz 1995). This is a frequent phenomenon that has been found in diverse taxa subject to varying levels of time stress (Yamahira and Conover 2002, Van Doorslaer and Stoks 2005b). Both field data (reviewed by Corbet et al. [2006]) and the life-cycle simulation support this interpretation, as the sampled populations of central species live close to the northern limit of a univoltine life cycle, indicating strong time constraints. Such a link between voltinism and countergradient variation was recently also shown in an interspecific study of a related taxon (Shama et al. 2011). In contrast to this, northern species showed lower growth rate than central and southern species. This is consistent with cogradient variation (Conover and Schultz 1995) and may reflect that a shift to semivoltinism drastically increases the time available for growth.

No species showed significantly decreased growth rates within the experimental thermal range (Fig. 4; Appendix E: Fig. E1), suggesting that optimal temperatures are reached above average environmental temperatures (Fig. 2), conflicting with the first prediction from the two optimality models. Mismatches between 
optimal and environmental temperature have commonly been observed. These mismatches have been suggested to be adaptive; because fitness decreases more rapidly at temperatures above the optimum, shifting the optimal temperature to temperatures above average conditions will be an optimal strategy to avoid the negative effects of exposures to extreme temperatures (Martin and Huey 2008). Alternatively, they may indicate limited evolvability of TPCs (Knies et al. 2009). This is an intriguing field of inquiry but we cannot test these ideas further without reliable estimates of thermal optima.

\section{Specialist-generalist}

Southern species clearly maintain near-maximal growth rates over a much wider range of experimental temperatures than the other species, suggesting they are thermal generalists (Fig. 4C). Regrettably, the limited range of experimental temperatures did not allow us to directly estimate the breadths of performance curves. Nevertheless, by focusing on the shape of the rising phase, one can still infer the general characteristics of species in this respect (Dell et al. 2011, Huey and Kingsolver 2011). In doing so, we assume that the experimental thermal range covers a substantial part of the initial, rising phase. Given that (1) all previous studies of growth rates in Coenagrion species have detected decreased growth rates within the temperature span of $24-27^{\circ} \mathrm{C}$ (Waringer and Humpesch 1984 , Krishnaraj and Pritchard 1995, Van Doorslaer and Stoks $2005 a, b)$; (2) that the increase in growth rates of $C$. mercuriale slow down toward the upper range of temperatures; and (3) that the experimental thermal range covers almost the full range of temperatures experienced by central and northern species, we consider it highly unlikely that the inclusion of higher experimental temperatures would reveal markedly increased growth rates above experimental temperatures in any of the studied species. In addition, the variation we observe in these reaction norms (from upward-convex to nearexponential) are consistent with the hypothesis that TPC shape during the initial phase largely follows Boltzmann-Arrhenius kinetics (Dell et al. 2011, but see Knies and Kingsolver 2010). Under this paradigm, the shallow slopes of southern species can be understood as reflecting low activation energies of growth rates in these species, indicative of selection for high performance over a broad range of temperatures, i.e., thermal generalization (Dell et al. 2011). We thus feel confident labeling the two southern species as thermal generalists compared to northern and central species (which display much steeper slopes, indicative of higher activation energies, restricting maximal growth rates to a narrower temperature interval). This interpretation directly conflicts with the prediction from Gilchrist's (1995) model, that southern species should be thermally specialized because they experience low relative levels of amonggeneration variation. Lynch and Gabriel's (1987) model, on the other hand, predicts that southern species should be the most generalized because they experience high levels of within-generation variability. This latter prediction is indeed supported under the previous interpretation of the data.

Reaction norms of northern species differ dramatically from the other species, being upward-concave within the experimental range of temperatures (Fig. 4A). Interestingly, the observed pattern indicates that northern species are thermal specialists, but with thermal optima above $21.5-24^{\circ} \mathrm{C}$, which, according to both modeled and empirical data, is expected to be temperatures they rarely experience in nature (Fig. 2; Appendix E: Fig. E1; Johansson and Norling 1994). Clearly, the location of these optima alone makes it evident that even when considering diapause and voltinism, our estimates of within-generation variability and average temperature are not sufficient to explain natural variation in thermal reaction norms of growth rates in these species. Several additional factors may be important shaping thermal sensitivities of growth rates and may be necessary to take into consideration. Some examples are: (1) thermal variation at smaller spatial and temporal scales; (2) constraints on the evolution of thermal responses; (3) the potential of organisms to acclimate to different temperatures; and (4) the possibility for more complex relationships between growth rates and fitness. We feel that a deeper understanding of each of these issues will help to further the study of thermal adaptation and we will therefore discuss them in some detail next.

\section{Thermal variation at a finer scale}

First, the coenagrionids studied here generally exploit areas close to the surface in the laboratory (Johansson 2000), but whether natural populations display genetic variation in this respect remains to be tested. If they do, this may alter the latitudinal patterns experienced in environmental variation. If, for example, individuals of northern species exploit much shallower microhabitats than their southern counterparts (either through individual movement or through parental site selection), they would experience higher temperatures and more variation than our simulations suggest (Jacobs et al. 1998), but whether this has the potential to explain the high thermal optima of northern species is not clear.

Latitudinal differences in diurnal temperature fluctuations may also be important. Because these are heavily influenced by lake-specific characteristics, the FLake model is not sufficient to explore variation at that fine scale. Nevertheless, our life-cycle simulation reliably predicts known voltinism patterns (Corbet et al. 2006), indicating that it is reasonably realistic. Voltinism patterns of southern species are still unknown (Dijkstra and Lewington 2006) and if they deviate from the predicted univoltine life cycle, patterns of among-towithin generation thermal variability would change. Further investigations of voltinism patterns and, ideally, field body temperatures would be very valuable. 


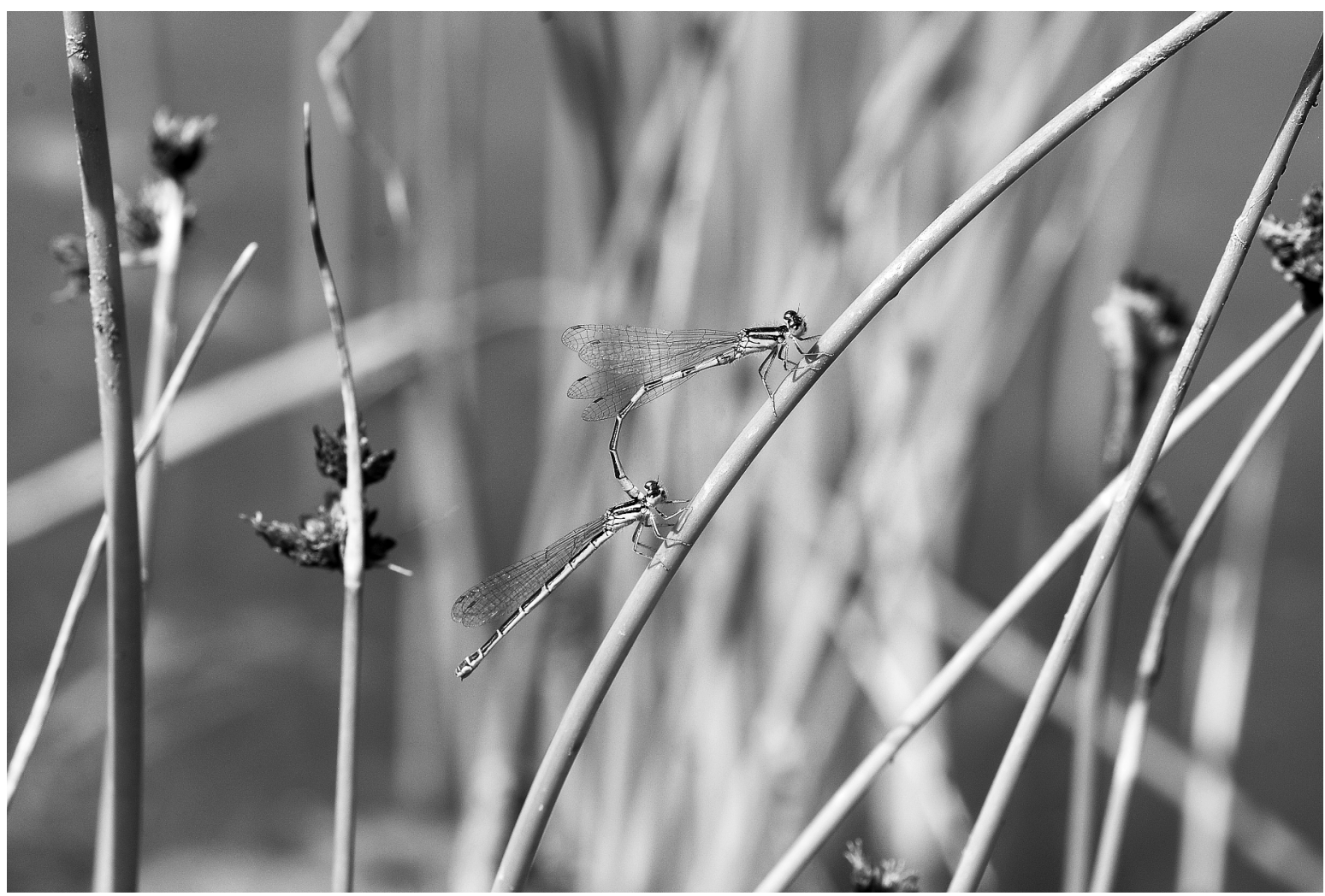

Plate 1. A mated pair of the damselfly Coenagrion scitulum, one of the studied species having a southern European distribution. Photo credit: Jochen Rodenkirchen.

\section{Evolutionary constraints}

Second, in interspecific comparisons, one compares taxa separated for an unknown, but relatively long, time and it cannot be known whether the observed difference reflects adaptation to present thermal regimes rather than reflecting adaptation to historical thermal regimes (or selective agents apart from temperature). We were not able to control for phylogenetic relationships among the six species studied here, as no complete species-level phylogeny is currently available. The two central species are closely related based on molecular data (Dumont et al. 2010), while the relationships between the four other species remain unresolved.

Differences in the amount of spatial variation experienced across species' ranges could also constrain the evolution of thermal reaction norms, if gene flow is high (Via and Lande 1985). Based on the life-cycle simulations, southern and central species experience nearly 2.5 times more variation in average temperature and nearly 10 times more variation in within-generation thermal variation across their range than northern species. We consider the decrease in spatial variation across the range of northern species an alternative explanation for the specialization observed in northern species, and we are currently investigating how intraspecific variation in growth rates relates to variation in the thermal environment experienced across the whole range of these species (V. Nilsson-Örtman, R. Stoks, M. De Block, and F. Johansson, unpublished manuscript).

\section{Thermal acclimation}

Third, the two optimality models explicitly assume that no acclimation occurs. Under certain conditions, some species may evolve very strong acclimation responses of a specialist reaction norm, instead of increasing the breadth of the reaction norm. Lynch and Gabriel's (1987) model has been extended to investigate when thermal acclimatization occurs (Gabriel and Lynch 1992), finding that increased amonggeneration thermal variability favors increased acclimation responses. If so, strong acclimation responses would be expected in central and northern species, implying that the relatively broad performance breadths of central species could be due either to large withingeneration thermal variability or strong acclimation responses (but it cannot explain why northern species grow so slow at low temperatures). Because we used constant temperature treatments, we cannot separate differences in the shape of reaction norms from differences in acclimation responses - the observed reaction norms are a combination of both (Huey and Berrigan 1996). An alternative approach is to measure acute thermal sensitivities (Kingsolver and Woods 1997) 
or use fluctuating temperatures (differing in average temperature). However, using fluctuating temperatures has recently been shown to have no effect on the rank order of life-history trait values compared to using constant temperature regimes (Fischer et al. 2011), but another recent study shows that they may have stronger effects on stress-resistance traits (Terblanche et al. 2010).

\section{Fitness links}

Fourth, although somatic growth is essential for all multicellular organisms and thus is intrinsically linked to fitness (Case 1978, Arendt 1997), the two models we tested assume that there is a single, unique function that determines how performance affects fitness. A large number of genes likely underlie a complex trait like growth rate and different sets of genes probably differ in how they contribute to fitness, making the true fitness function a mixture of additive and multiplicative effects (Angilletta et al. 2006). The fitness consequences of a given thermal strategy will also depend, for example, on the thermal characteristics of predators present (Huey and Slatkin 1976). The need for a "game theory of thermal coadaptation" has been called for (Angilletta et al. 2006) and is slowly emerging, providing novel hypotheses regarding the evolution of TPC shape (Mitchell and Angilletta 2009).

Certain aspects of fitness may nonetheless remain fixed across latitudes. For example, we consider it likely that the benefits gained from completing more generations in a given time, or having an increased chance of survival early in larval life (Suhling and Lepkojus 2001) are likely to remain relatively fixed. Because development rates generally increase more rapidly with elevated temperatures than do growth rates, adults typically emerge smaller at higher temperatures (Kingsolver and Huey 2008). At any given temperature, however, fastgrowing individuals still mature at a relatively larger adult size. Because size and fecundity are typically correlated, this aspect of fitness may also remain relatively constant.

\section{Emerging insights and perspectives}

The results from the life-cycle simulation and the laboratory experiment together challenge the common notion that temperate environments can be regarded as simply "thermally heterogeneous" and that organisms inhabiting them should be thermal generalists. Instead, we have shown how diapause and voltinism can act to buffer how environmental variation is experienced by individuals, making thermal specialization optimal in high-latitude environments under certain conditions (without these mechanisms, high-latitude environments would be very inhospitable to thermal specialists). The life-cycle simulations indicate that two separate mechanisms might favor a reduction in TPC breadth in organisms with flexible life cycles inhabiting highlatitude environments. First, an increased life span makes the thermal environment increasingly similar from generation to generation as the among-year thermal variation is averaged out over the progressively longer lifetime of individuals (note, however, that in this case, this seems to occur at latitudes above those from which our northern species were sampled). Second, species with a temperature-induced diapause will experience less variation around the average temperature within the growth season toward higher latitudes.

Clear examples of geographical trends in thermal specialization are rare in the literature, but our results can be compared to several cases where tropical species or populations have been found to be more thermally specialized than temperate counterparts (e.g., Cunningham and Read 2002, Eggert et al. 2003, Latimer et al. 2011). These cases also lend apparent support to Lynch and Gabriel's (1987) prediction that generalists are to be expected when within-generation variability is large, suggesting that this pattern may be of wider importance. Together, our results reinforce the current view that we must not only consider environmental variation per se, but must consider how environmental variation interacts with species' life histories (Ragland and Kingsolver 2007, Cooper et al. 2010) and that we must focus on patterns in thermal variation rather than on differences in average conditions (Clusella-Trullas et al. 2011). Nevertheless, the upward-concave reaction norms of northern species clearly cannot be sufficiently explained at present without considering additional factors. Future studies that quantify environmental variation with respect to generation length and other life history characteristics will surely provide many valuable insights into the factors responsible for shaping species' thermal characteristics.

\section{ACKNOWLEDGMENTS}

V. Nilsson-Örtman, R. Stoks, M. De Block, and F. Johansson conceived the study and performed the laboratory experiment; V. Nilsson-Örtman developed the computer model, analyzed data, and wrote the paper; and R. Stoks, M. De Block, and F. Johansson contributed to writing. M. De Block is a postdoctoral fellow of the Fund for Scientific ResearchFlanders (FWO). Financial support to R. Stoks came from FWO and the KULeuven research fund and to V. NilssonÖrtman and F. Johansson from the Swedish Research Council Formas. We thank Owen Rowe, Helena Johansson, Michael Angilletta Jr., and two anonymous reviewers for commenting on a previous version of the manuscript. We also thank Szymon Sniegula for data on degree-day requirements, and all those who have helped us to locate suitable sampling sites.

\section{Literature Cited}

Angilletta, M. J., Jr. 2009. Thermal adaptation: a theoretical and empirical synthesis. Oxford University Press, Oxford, UK.

Angilletta, M. J., Jr., A. F. Bennett, H. Guderley, C. A. Navas, F. Seebacher, and R. S. Wilson. 2006. Coadaptation: a unifying principle in evolutionary thermal biology. Physiological and Biochemical Zoology 79:282-294.

Arendt, J. D. 1997. Adaptive intrinsic growth rates: an integration across taxa. Quarterly Review of Biology 72:149-177.

Askew, R. R. 2004. The dragonflies of Europe. Revised edition. Harley Books, Colchester, UK. 
Brown, J. H., J. F. Gillooly, A. P. Allen, V. M. Savage, and G. B. West. 2004. Toward a metabolic theory of ecology. Ecology 85:1771-1789.

Case, T. J. 1978. On the evolution and adaptive significance of postnatal growth rates in the terrestrial vertebrates. Quarterly Review of Biology 53:243-282.

Clusella-Trullas, S., T. M. Blackburn, and S. L. Chown. 2011. Climatic predictors of temperature performance curve parameters in ectotherms imply complex responses to climate change. American Naturalist 177:738-751.

Conover, D. O., and E. T. Schultz. 1995. Phenotypic similarity and the evolutionary significance of countergradient variation. Trends in Ecology and Evolution 10:248-252.

Cooper, B. S., M. Czarnoleski, and M. J. Angilletta, Jr. 2010. Acclimation of thermal physiology in natural populations of Drosophila melanogaster: a test of an optimality model. Journal of Evolutionary Biology 23:2346-2355.

Corbet, P. S. 1999. Dragonflies: behaviour and ecology of Odonata. Cornell University Press, Ithaca, New York, USA.

Corbet, P. S., F. Suhling, and D. Soendgerath. 2006. Voltinism of Odonata: a review. International Journal of Odonatology 9:1-44.

Cunningham, S., and J. Read. 2002. Comparison of temperate and tropical rainforest tree species: photosynthetic responses to growth temperature. Oecologia 133:112-119.

De Block, M., S. Slos, F. Johansson, and R. Stoks. 2008. Integrating life history and physiology to understand latitudinal size variation in a damselfly. Ecography 31:115123.

Dell, A. I., S. Pawar, and V. M. Savage. 2011. Systematic variation in the temperature dependence of physiological and ecological traits. Proceedings of the National Academy of Sciences USA 108:10591-10596.

Demont, M., W. U. Blanckenhorn, D. J. Hosken, and T. W. J. Garner. 2008. Molecular and quantitative genetic differentiation across Europe in yellow dung flies. Journal of Evolutionary Biology 21:1492-1503.

Dijkstra, K. D. B., and R. Lewington. 2006. Field guide to the dragonflies of Britain and Europe. British Wildlife Publishing, Gillingham, Dorset, UK.

Dumont, H. J., A. Vierstraete, and J. R. Vanfleteren. 2010. A molecular phylogeny of the Odonata (Insecta). Systematic Entomology 35:6-18.

Eggert, A., E. M. Burger, and A. M. Breeman. 2003. Ecotypic differentiation in thermal traits in the tropical to warmtemperate green macrophyte Valonia utricularis. Botanica Marina 46:69-81.

Fischer, K., N. Kölzow, H. Höltje, and I. Karl. 2011. Assay conditions in laboratory experiments: Is the use of constant rather than fluctuating temperatures justified when investigating temperature-induced plasticity? Oecologia 166:22-33.

Gabriel, W., and M. Lynch. 1992. The selective advantage of reaction norms for environmental tolerance. Journal of Evolutionary Biology 5:41-59.

Gilchrist, G. W. 1995. Specialists and generalists in changing environments. I. Fitness landscapes of thermal sensitivity. American Naturalist 146:252-270.

Huey, R. B., and D. Berrigan. 1996. Testing evolutionary hypotheses of acclimation. Pages 205-237 in I. A. Johnston and A. F. Bennett, editors. Animals and temperature: phenotypic and evolutionary adaptation. Cambridge University Press, Cambridge, UK.

Huey, R. B., and J. G. Kingsolver. 2011. Variation in universal temperature dependence of biological rates. Proceedings of the National Academy of Sciences USA 108:10377-10378.

Huey, R. B., and M. Slatkin. 1976. Cost and benefits of lizard thermoregulation. Quarterly Review of Biology 51:363-384.

Irlich, U. M., J. S. Terblanche, T. M. Blackburn, and S. L. Chown. 2009. Insect rate-temperature relationships: environmental variation and the metabolic theory of ecology. American Naturalist 174:819-835.
Izem, R., and J. G. Kingsolver. 2005. Variation in continuous reaction norms: quantifying directions of biological interest. American Naturalist 166:277-289.

Jacobs, A. F. G., B. G. Heusinkveld, and J. P. Nieveen. 1998. Temperature behavior of a natural shallow water body during a summer period. Theoretical and Applied Climatology 59:121-127.

Johansson, F. 2000. The slow-fast life style characteristics in a suite of six species of odonate larvae. Freshwater Biology 43:149-159.

Johansson, F., and U. Norling. 1994. A five year study of the larval life history of Coenagrion hastulatum (Charpentier) and C. armatum (Charpentier) in northern Sweden (Zygoptera: Coenagrionidae). Odonatologica 23:355-364.

Kingsolver, J. G. 2009. The well-temperatured biologist. American Naturalist 174:755-768.

Kingsolver, J. G., and R. Huey. 2008. Size, temperature, and fitness: three rules. Evolutionary Ecology Research 10:251268.

Kingsolver, J. G., and H. A. Woods. 1997. Thermal sensitivity of growth and feeding in Manduca sexta caterpillars. Physiological and Biochemical Zoology 70:631-638.

Knies, J. L., and J. G. Kingsolver. 2010. Erroneous Arrhenius: modified Arrhenius model best explains the temperature dependence of ectotherm fitness. American Naturalist 176:227-233.

Knies, J. L., J. G. Kingsolver, and C. L. Burch. 2009. Hotter is better and broader: thermal sensitivity of fitness in a population of bacteriophages. American Naturalist 173:419-430.

Krishnaraj, R., and G. Pritchard. 1995. The influence of larval size, temperature, and components of the functional response to prey density on growth rates of the dragonflies Lestes disjunctus and Coenagrion resolutum (Insecta: Odonata). Canadian Journal of Zoology 73:1672-1680.

Lake Model FLake. 2009. FLake online. http://www.flake. igb-berlin.de/index.shtml

Latimer, C. A. L., R. S. Wilson, and S. F. Chenoweth. 2011. Quantitative genetic variation for thermal performance curves within and among natural populations of Drosophila serrata. Journal of Evolutionary Biology 24:965-975.

Lynch, M., and W. Gabriel. 1987. Environmental tolerance. American Naturalist 129:283-303.

Martin, T. L., and R. B. Huey. 2008. Why "suboptimal" is optimal: Jensen's inequality and ectotherm thermal preferences. American Naturalist 171:102-118.

Mitchell, W. A., and M. J. Angilletta, Jr. 2009. Thermal games: frequency dependent models of thermal adaptation. Functional Ecology 23:510-520.

Navas, C. A. 1996. Metabolic physiology, locomotor performance, and thermal niche breadth in neotropical anurans. Physiological Zoology 69:1481-1501.

Norling, U. 1984. Life history patterns in the northern expansion of dragonflies. Advances in Odonatology 2:127156.

Pöykkö, H., and T. Tammaru. 2010. Countergradient vs. cogradient variation in growth and diapause in a lichenfeeding moth, Eilema depressum (Lepidoptera: Arctiidae). Journal of Evolutionary Biology 23:1278-1285.

R Development Core Team. 2008. R: a language and environment for statistical computing. R Foundation for Statistical Computing, Vienna, Austria.

Ragland, G. J., and J. G. Kingsolver. 2007. Influence of seasonal timing on thermal ecology and thermal reaction norm evolution in Wyeomyia smithii. Journal of Evolutionary Biology 20:2144-2153.

Roff, D. A. 2002. Life history evolution. Sinauer Associates, Sunderland, Massachusetts, USA.

Rose, K. E., R. L. Atkinson, L. A. Turnbull, and M. Rees. 2009. The costs and benefits of fast living. Ecology Letters 12:1379-1384. 
Shama, L. N. S., M. Campero-Paz, M. Wegner, M. De Block, and R. Stoks. 2011. Latitudinal and voltinism compensation shape thermal reaction norms for growth rate. Molecular Ecology 20:2929-2941.

Simmons, A., S. M. Uppala, D. Dee, and S. Kobayashi. 2007. ERA-interim: new ECMWF reanalysis products from 1989 onwards. ECMWF Newsletter 110:25-35.

Śniegula, S., F. Johansson, and V. Nilsson-Örtman. In press. Differentiation in developmental rate across geographic regions: a photoperiod driven latitude compensating mechanism? Oikos.

Stoks, R., and M. De Block. 2011. Rapid growth reduces cold resistance: evidence from latitudinal variation in growth rate, cold resistance and stress proteins. PLoS ONE 6:e16935.

Strobbe, F., and R. Stoks. 2004. Life history reaction norms to time constraints in a damselfly: differential effects on size and mass. Biological Journal of the Linnean Society 83:187-196.

Suhling, F., and S. Lepkojus. 2001. Differences in growth and behaviour influence asymmetric predation among earlyinstar dragonfly larvae. Canadian Journal of Zoology 79:854-860.

Terblanche, J. S., C. Nyamukondiwa, and E. Kleynhans. 2010. Thermal variability alters climatic stress resistance and plastic responses in a globally invasive pest, the Mediterranean fruit fly (Ceratitis capitata). Entomologia Experimentalis et Applicata 137:304-315.

Van Doorslaer, W., and R. Stoks. 2005a. Growth rate plasticity to temperature in two damselfly species differing in latitude: contributions of behaviour and physiology. Oikos 111:599605.

Van Doorslaer, W., and R. Stoks. 2005b. Thermal reaction norms in two Coenagrion damselfly species: contrasting embryonic and larval life-history traits. Freshwater Biology 50:1982-1990.

Via, S., and R. Lande. 1985. Genotype-environment interaction and the evolution of phenotypic plasticity. Evolution 39:505-522.

Waringer, J. A., and U. H. Humpesch. 1984. Embryonic development, larval growth and life cycle of Coenagrion puella (Odonata: Zygoptera) from an Austrian pond. Freshwater Biology 14:385-399.

Yamahira, K., and D. Conover. 2002. Intra- vs. interspecific latitudinal variation in growth: adaptation to temperature or seasonality? Ecology 83:1252-1262.

\section{Supplemental Material}

\section{Appendix A}

Full description of the FLake model and larval life-cycle simulations (Ecological Archives E093-118-A1).

\section{Appendix B}

Detailed description of the procedure used for rearing Coenagrion larvae (Ecological Archives E093-118-A2).

\section{Appendix C}

Description of the missing data imputation procedure (Ecological Archives E093-118-A3).

\section{Appendix D}

Description of the calculations of size-corrected relative growth rates (Ecological Archives E093-118-A4).

\section{Appendix E}

Figures and tables showing among-family variation in reaction norms of growth rates, a full list of collecting localities, and the result from the species-specific ANOVAs on the shape of TPCs (Ecological Archives E093-118-A5).

\section{Supplement}

Stand-alone R code for running the larval life-cycle simulations with dummy data (Ecological Archives E093-118-S1). 